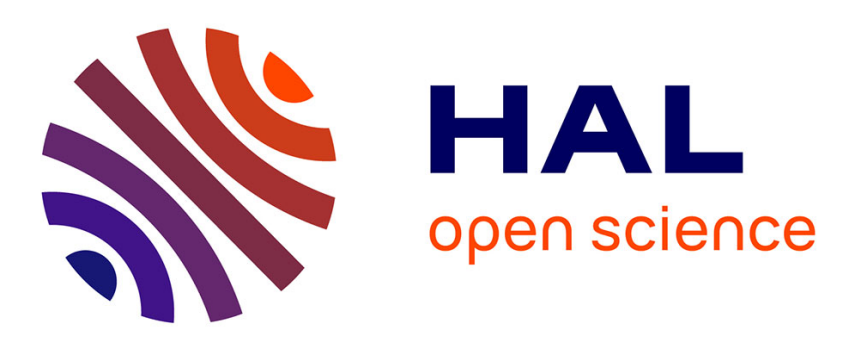

\title{
Electronic structure and hydration of tetramine cobalt hydride complexes.
}

\author{
Anirban Bhattacharjee, Alexander K. H. Weiss, Vincent Artero, Martin J \\ Field, Thomas S. Hofer
}

\section{To cite this version:}

Anirban Bhattacharjee, Alexander K. H. Weiss, Vincent Artero, Martin J Field, Thomas S. Hofer. Electronic structure and hydration of tetramine cobalt hydride complexes.. Journal of Physical Chemistry B, 2014, 118 (20), pp.5551-5561. 10.1021/jp502651s . hal-01069163

\section{HAL Id: hal-01069163 https://hal.science/hal-01069163}

Submitted on 29 May 2018

HAL is a multi-disciplinary open access archive for the deposit and dissemination of scientific research documents, whether they are published or not. The documents may come from teaching and research institutions in France or abroad, or from public or private research centers.
L'archive ouverte pluridisciplinaire HAL, est destinée au dépôt et à la diffusion de documents scientifiques de niveau recherche, publiés ou non, émanant des établissements d'enseignement et de recherche français ou étrangers, des laboratoires publics ou privés. 


\title{
Electronic Structure and Hydration of Tetramine Cobalt Hydride Complexes
}

\author{
Anirban Bhattacharjee, ${ }^{\dagger}$ Alexander K. H. Weiss, ${ }^{\ddagger}$ Vincent Artero, "Martin J. \\ Field, ${ }^{*, \dagger}$ and Thomas S. Hofer, ${ }^{*,}$ \\ DYNAMO/DYNAMOP, Institut de Biologie Structurale (UMR5075, CEA, CNRS, \\ Université Grenoble I), 6 rue Jules Horowitz, 38000 Grenoble, France, Department of \\ Theoretical Chemistry, Institute of General, Inorganic and Theoretical Chemistry, \\ University of Innsbruck, Inrain 80-82, A-6020 Innsbruck, Austria, and Laboratoire de \\ Chimie et Biologie des Métaux (UMR 5249, CNRS, CEA,Université Grenoble I), \\ DSV/iRTSV, CEA-Grenoble, 17 rue des Martyrs F-38054 Grenoble Cedex 9, France \\ E-mail: martin.field@ibs.fr; t.hofer@uibk.ac.at
}

${ }^{*}$ To whom correspondence should be addressed

${ }^{\dagger}$ DYNAMO

${ }^{\ddagger}$ Innsbruck

${ }^{\top} \mathrm{LCBM}$ 


\begin{abstract}
In this work we have studied two hydrido-tetramine-cobalt(III) complexes using a mixture of computational techniques. These species were chosen as simple and computationally tractable models of the $\mathrm{Co}$ (III)-hydrido compounds that are known to be important intermediates in the catalytic cycles of hydrogen evolution mediated by the cobaloxime complexes. We have performed both static density functional theory (DFT) calculations of the complexes in implicit solvent and adaptive hybrid DFT/molecular mechanical (MM) molecular dynamics (MD) simulations in explicit solvent, and compared our results to the experimental structural and spectral data that is available for one of the compounds. A principal aim of the study has been to provide a benchmark for future work on cobaloxime and other hydrogen-evolving catalysts using adaptive DFT/MM MD methods.
\end{abstract}

\title{
Keywords
}

Cobalt hydrides, density functional theory, electronic structure, molecular dynamics simulations, qc/mm potentials

\section{Introduction}

Hydrogen evolution is a key process in the generation of fuel from solar energy, and much research has gone into the design of cheap and robust catalysts that can carry out this reaction. In this context, cobaloxime complexes containing the relatively abundant metal, cobalt, are among the most efficient molecular catalysts that are known. ${ }^{1,2}$ It is now generally accepted that the catalytic cycle for hydrogen evolution from these species proceeds via protonation of the $\mathrm{Co}(\mathrm{I})$ species,${ }^{3-15}$ yielding a $\mathrm{Co}(\mathrm{III})$-hydrido-cobaloxime $(\mathrm{Co}(\mathrm{III})-\mathrm{H})$ intermediate that, after further reduction to the $\mathrm{Co}(\mathrm{II})-\mathrm{H}$ state, ${ }^{5,16-18}$ can evolve dihydrogen through either protonation of the hydride moiety or bimolecular reductive elimination. 
In recent work, we have been interested in the mechanism of hydrogen evolution from a number of cobaloxime complexes and in the investigation of the intermediates that occur during their catalytic cycles. In one study, we characterized, using a mixture of experiment and theory, the crucial hydrido-cobaloxime compound that is central to hydrogen-evolution. ${ }^{19,20}$ The theoretical part of this work employed density functional theory (DFT) quantum chemical (QC) calculations with an implicit model of solvent. Such methods can give an accurate description of electronic structure, reactivity and other aspects of a complex, but they are essentially static and neglect potentially-important dynamical effects, either within the complex itself or between it and the surrounding solvent molecules.

DFT methods are probably the minimum level of QC theory that is necessary to obtain a reasonable description of transition-metal complexes. However, molecular dynamics (MD) simulations of sufficient length with pure DFT potentials are computationally very challenging and limited to quite small numbers of atoms. A viable alternative is to employ mixed DFT/molecular mechanical (MM) potentials that represent the complex and a small number of surrounding solvent molecules quantum chemically and treat the rest of the system with a simpler MM force-field approach.

In the current work, we employ a hybrid DFT/MM potential in an initial investigation of the dynamics and solvent structure of hydrido-Co(III) compounds. To start with, we have chosen to simulate two smaller hydrido-tetramine-cobalt(III) complexes, instead of the larger and more complicated cobaloxime compounds. The simulated complexes, which we denote by $\left[\mathrm{HCo}(\mathrm{en})_{2}\right]^{+}$and $\left[\mathrm{HCo}(\text { tmen })_{2}\right]^{+}$, are shown schematically in Figure 1 . We note Figure 1 that the methylated tetramine complex, $\left[\mathrm{HCo}(\text { tmen })_{2}\right]^{+}$, was synthesized by Rae et al. ${ }^{21}$ is surprisingly stable, and is one of the few cobalt hydrides for which a crystal structure and full spectroscopic information in water exists. It therefore serves as a reference to which future studies of larger cobalt-complexes and other transition metal hydrides can be compared.

The outline of the paper is as follows. Section describes the methods that we have used to simulate the complexes and to analyse the results, section discusses the results of our 
calculations, and section concludes.

\section{Methods}

We performed pure QC calculations on the complexes using DFT and more sophisticated ab initio methods, as well as hybrid potential QC/MM simulations within the quantum mechanical charge field molecular dynamics (QMCF-MD) approximation. We describe each in turn.

\section{Electronic Structure Calculations}

Standalone QC calculations were carried out with the ORCA quantum chemical program. ${ }^{22}$ For the DFT and time-dependent DFT (TDDFT) calculations, we employed both the BP86 ${ }^{23,24}$ and B3LYP ${ }^{25,26}$ functionals, along with an Ahlrichs TZVP basis set. ${ }^{27}$ The combination of the BP86 functional and triple- $\zeta$ quality basis sets has been shown to perform well for other cobalt complexes, such as the corrinoids in their ground and excited states. ${ }^{28-31}$ In our previous article, we also found that the B3LYP functional, with a TZVP basis, produces good transition energies for the hydrido tetramine compound using the TDDFT formalism. Solvent effects were included using the implicit COSMO model for water. ${ }^{32}$

To complement the DFT calculations, we also employed an ab initio CASSCF method. Due to computational expense we employed a TZVP basis for the cobalt, hydride and nitrogen atoms and a smaller SVP basis for the remaining atoms. In itself, the CASSCF method is not adequate to describe dynamic correlation, so it is common to supplement it with a perturbative correction, such as CASPT2. ${ }^{28}$ We chose the strongly contracted version of NEVPT2 $2^{33,34}$ that has been implemented in the ORCA program and has an advantage of being free from intruder states. An alternative approach would be to employ a MRCI method, although these are computationally very demanding even for complexes of the size that we are studying. We have preferred, therefore, to leave these types of calculations for 
the future.

\section{The QMCF-MD Methodology}

The QMCF-MD methodology is an adaptive QC/MM approach that has been used extensively for the study of solvated species. We describe it only briefly here as a number of detailed reports and reviews on the approach already exist. Readers are referred to references $^{35-39}$ for further information.

In a QMCF-MD simulation, the system is partitioned into a $\mathrm{QC}$ and an MM region. The QC region is further subdivided into a central core, which contains the solute and some solvent molecules, and an external "intermediate" layer that contains only solvent species. The size of the intermediate layer is such that all non-Coulombic interactions between the solute species in the core and the solvent molecules in the MM region can be neglected. As a result, the particles within the core zone are affected only by forces from particles within the $\mathrm{QC}$ region, $F^{\mathrm{QC}}$, and the Coulombic terms between $\mathrm{QC}$ and $\mathrm{MM}$ atoms:

$$
F_{q}^{\text {Core }}=F_{q}^{\mathrm{QC}}+\sum_{m=1}^{M} \frac{q_{m} q_{q}}{r_{m q}^{2}}\left[1+2\left(\frac{\epsilon+1}{2 \epsilon-1}\right)\left(\frac{r_{m q}}{r_{c}}\right)^{3}\right] \frac{r_{m q}}{\left\|r_{m q}\right\|}
$$

In this equation, the subscripts $m$ and $q$ refer to MM and QC particles, respectively, $q$ is an atomic charge, $r_{m q}$ is an internuclear distance, $\epsilon$ is the permittivity of water and $M$ is the number of atoms in the MM region within the cutoff range, $r_{c}$. The charges on the MM atoms are fixed throughout the simulation and are determined by the force field that is being used. By contrast, the charges on the QC atoms are redetermined at every MD step using a population analysis, thereby accounting for the geometrical changes that occur during the dynamics. We have previously shown that the use of Mulliken populations ${ }^{40}$ and a Dunning DZP basis set ensures compatibility between the charges on the QC atoms and those of the MM force field. ${ }^{41-44}$ The last part of the expression in square-brackets results from the application of a reaction-field correction ${ }^{45}$ that adjusts for the error introduced by 
the Coulombic cutoff.

The force expression with the QC intermediate layer is identical to that of the core region, except that there are in addition non-Coulombic coupling contributions, $F_{m q}^{\mathrm{nC}}$ :

$$
F_{q}^{\text {Layer }}=F_{q}^{\mathrm{QC}}+\sum_{m=1}^{M} \frac{q_{m} q_{q}}{r_{m q}^{2}}\left[1+2\left(\frac{\epsilon+1}{2 \epsilon-1}\right)\left(\frac{r_{m q}}{r_{c}}\right)^{3}\right] \frac{r_{m q}}{\left\|r_{m q}\right\|}+F_{m q}^{\mathrm{nC}}
$$

Particles in the MM region of the simulation box experience forces according to:

$$
F_{m}^{\mathrm{MM}}=\sum_{m, n \text { pairs }}^{M} F_{m n}^{\mathrm{MM}}+\sum_{q=1}^{Q_{1}+Q_{2}} \frac{q_{m} q_{q}}{r_{m q}^{2}}\left[1+2\left(\frac{\epsilon+1}{2 \epsilon-1}\right)\left(\frac{r_{m q}}{r_{c}}\right)^{3}\right] \frac{r_{m q}}{\left\|r_{m q}\right\|}+\sum_{q=1}^{Q_{2}} F_{m q}^{n C}
$$

where $Q_{1}$ and $Q_{2}$ are the number of $\mathrm{QC}$ atoms in the the core and the intermediate layer regions of the $\mathrm{QC}$ region, respectively, and the term $F_{m n}^{\mathrm{MM}}$ indicates the force between the pair of MM atoms $m$ and $n$.

To ensure a continuous transition with respect to exchange of solvent molecules between the QC and MM regions, a smoothing function is applied to the forces of the atoms at the boundary of the intermediate layer. The forces on these atoms has the form:

$$
F_{q}^{\text {Smooth }}=S(r) *\left(F_{q}^{\text {Layer }}-F_{q}^{\mathrm{MM}}\right)+F_{q}^{\mathrm{MM}}
$$

where $F_{q}^{\text {Layer }}$ is the force for the atom given in equation $2, F_{q}^{\mathrm{MM}}$ is the force calculated as if the atom were in the MM region, and the smoothing function, applied between the distances $r_{\min }$ and $r_{\max }$, is:

$$
S(r)= \begin{cases}1 & r \leq r_{\min } \\ \frac{\left(r_{\max }^{2}-r^{2}\right)^{2}\left(r_{\max }^{2}+2 r^{2}-3 r_{\min }^{2}\right)}{\left(r_{\max }^{2}-r_{\min }^{2}\right)^{3}} & r_{\min }<r \leq r_{\max } \\ 0 & r>r_{\max }\end{cases}
$$




\section{Simulations}

The two Co(III)-hydride complexes that we simulated were placed in cubic simulation boxes with 2000 water molecules, giving box lengths of 39.2 and $39.3 \AA$ for the $\left[\mathrm{HCo}(\mathrm{en})_{2}\right]^{+}$and $\left[\mathrm{HCo}(\text { tmen })_{2}\right]^{+}$complexes, respectively. Initial simulations of several hundred picoseconds and at constant temperature and pressure were done with an MM force-field using generalised Amber Force Field (GAFF) ${ }^{46}$ force field parameters.

Following this initial equilibration phase, the QMCF-MD methodology was applied. Atoms in the QC region were treated at the DFT level of theory using the BP86 functional along with the 6-31G(d,p) basis for the remaining atoms. Diffuse basis functions were not included because they substantially increase the computation time without markedly affecting the results. The radii of the core, intermediate layer and smoothing zones were set

to $0.0-2.0 \AA, 2.0-7.0 \AA$ and $6.8-7.0 \AA$, respectively. For the atoms in the MM region, the flexible SPC-mTR ${ }^{47}$ water was employed. Charges on the atoms in the QC region, necessary for the $\mathrm{QC} / \mathrm{MM}$ Coulombic interactions, were obtained via Mulliken analysis at each MD timestep and a long-range reaction-field correction $^{48}$ was applied to the same interactions using a water permittivity of 78.3. Simulations were performed at $298 \mathrm{~K}$ using Berendsen's constant temperature algorithm ${ }^{49}$ with a timestep of 0.2 fs for a duration of $25 \mathrm{ps}$, of which the first 5 ps were taken to be equilibration. To give an idea of the time required for these simulations, they each took approximately six months of computer time running in parallel on an eight-core machine using the Turbomole package as the engine for the QC calculations. ${ }^{50}$

\section{Structural Analyses}

A range of standard structural analyses were performed, including the determination of radial (RDF) and angular (ADF) distribution functions. In addition, we employed the SLICE formalism ${ }^{39,51}$ to obtain three-dimensional distributions giving a detailed picture of the hydration of the complexes. For such analyses, the simulation trajectories were superimposed 
by aligning the solute to a predefined reference geometry.

The SLICE method can be seen as the equivalent of computer tomography imaging applied to a simulation trajectory. Planes parallel to a predefined plane are first employed to segment the system and then the positions of selected target atoms are projected onto these planes. An atom's contribution to the density distribution is then computed via a weighting function:

$$
w(i, j)=e^{-\alpha_{i} *\left(r_{i}-r_{j}\right)^{2}}
$$

where $r_{i}$ denotes the coordinate of a target atom and $r_{j}$ is the corresponding point on the plane. $\alpha_{i}$ is chosen empirically and determines how quickly the exponential function decays.

SLICE analysis yields much more information than spherical analysis schemes because of its use of a number of planes to collect projected data. A simplified version of SLICE is the two-dimensional particle mapping method ${ }^{51}$ in which the reference plane is defined with respect to two bond vectors issuing from a common atom. Taking an $\mathrm{NH}_{2}$ group as an example, the plane would pass through the $\mathrm{N}$ atom and have as its normal the cross-product of the two $\mathrm{N}-\mathrm{H}$ bond vectors.

\section{Laguerre Tessellation and Dynamics}

To analyze hydration layers around the solute, a space decomposition approach employing Laguerre tessellation ${ }^{52,53}$ was implemented using the Voro $++^{52}$ library. In this method, the Voronoi decomposition ${ }^{54}$ for indistinguishable particles is extended to describe distinguishable atoms using standard van der Waals radii as the distinguishing descriptors. ${ }^{55}$ The analysis proceeds by centring the simulation boxes at the Co atoms and identifying individual layers of solvent hydration around the complexes - rather like the layers of an onion. Such layers are similar to hydration shells obtained from pair distribution functions, although small differences may occur due to the different definitions of nearest neighbours.

Once the hydration layers have been identified, further analysis was applied to obtain 
layer-specific properties. In this work, we employed a mean residence time (MRT) analysis with direct counting ${ }^{56}$ to describe the residence time of water molecules within distinct layers. We chose a window spacing of $t^{*}=0.5$ ps to reflect average hydrogen-bond lifetime, which has been reported to be of this magnitude. ${ }^{57,58}$ The number of attempts to achieve one lasting exchange event for a given time window (i.e. $t^{*}=0.5 \mathrm{ps}$ ), with respect to a window size of $t^{*}=0.0 \mathrm{ps}$, when all displacements are taken into account, is described by the ratio of exchange $\left(R_{\text {ex }}\right)$ value:

$$
R_{\mathrm{ex}}=\frac{N_{\mathrm{ex}}^{t}}{N_{\mathrm{ex}}^{t^{*}}}
$$

To analyze residence times within distinct hydration layers we modified an approach developed by Chanda et al. for analyzing hydrogen-bond properties. ${ }^{59}$ Their method defines two time correlation functions (TCFs) as follows:

$$
\begin{aligned}
& C(t)=\frac{<h(0) h(t)>}{<h>} \\
& S(t)=\frac{<h(0) H(t)>}{<h>}
\end{aligned}
$$

Here $C(t)$ is the intermittent hydrogen-bond time correlation function, which represents the structural relaxation of hydrogen-bonds (including breaking and re-forming events), whereas $S(t)$ is the continuous hydrogen bond time correlation, which provides a strict definition of their lifetime. The function $h(t)$ is unity when a particular pair of sites is hydrogen-bonded at time $t$ and zero otherwise, whereas $H(t)$ is unity only if the pair of sites is continuously bonded between the times 0 and $t$.

In our version of the analysis, we redefine the functions $h(t)$ and $H(t)$ to reflect the presence of a molecule in a particular hydration layer, rather than the occurrence of a particular hydrogen-bond. With this modification, the $C(t)$ and $S(t)$ functions now measure intermittent and continuous hydration layer occupations, respectively. To aid in their interpretation 
we also fit the $C(t)$ and $S(t)$ functions to double-exponential expressions of the form:

$$
y=a \exp \left(-t / \tau_{1}\right)+(1-a) \exp \left(-t / \tau_{\mathrm{s}}\right)
$$

where the $\tau$ values are the long (l) and short (s) time contributions to the correlation function, respectively.

\section{Results and Discussion}

\section{Electronic Structure Calculations of $\left[\mathrm{HCo}(\mathrm{tmen})_{2}\right]^{+}$}

For the pure QC calculations, we focused upon the methylated $\left[\mathrm{HCo}(\operatorname{tmen})_{2}\right]^{+}$complex for which experimental data exists. The crystal structure of $\left[\mathrm{HCo}(\mathrm{tmen})_{2}\right]^{+}$from the X-ray diffraction study ${ }^{21}$ was used as the starting point for our study. Hydrogens were added and the complex was geometry optimized at the DFT level of theory using an implicit solvent model of water. Note that the cobalt has, in addition to the hydride, a water molecule ligand that was treated explicitly. Agreement between the experimental and theoretical structures is good with the $\mathrm{Co}-\mathrm{H}$ bond length after optimization being found to be $1.44 \AA$. The BP86 and B3LYP functionals produce very similar geometries with both the Ahlrichs TZVP basis and the larger, hybrid cc-pVTZ/TZVP set.

After these initial calculations we concentrated on an investigation of the spectra using

both TDDFT and ab initio methods. A summary of these results is shown in Table 1. Table 1 Overall, the performance of the TDDFT method is good. The higher energy band at $340 \mathrm{~nm}$ is well reproduced by all the methods. Agreement for the lower energy band at $444 \mathrm{~nm}$ is also satisfactory, with the B3LYP functional being slightly better than BP86 and, interestingly, the smaller 6-31G(d,p) basis better than TZVP.

If we consider the B3LYP/TZVP spectrum, the experimental band at $444 \mathrm{~nm}$ corresponds to a superposition of two approximately equally intense transitions at 415 and $418 \mathrm{~nm}$, 
whereas the band at $340 \mathrm{~nm}$ is comprised of an intense band at $326 \mathrm{~nm}$ and a less intense one at $322 \mathrm{~nm}$. All these bands involve transitions between the HOMO and HOMO-1, which are of metal d-type, and the LUMO and LUMO+1. The LUMO is mainly a $\sigma$ anti-bonding type orbital between metal d- and donor nitrogen p-orbitals in the equatorial plane, whereas the LUMO+1 is the $\sigma$ anti-bonding orbital between the axial hydrido ligand and metal dorbitals. These orbitals are illustrated in Figure 2. The lower energy transitions at 415 and $418 \mathrm{~nm}$ are dominated by transitions of type $\mathrm{Co}(\mathrm{d})$ to $\mathrm{Co}(\mathrm{d})-\mathrm{N}(\mathrm{p}) \sigma$ anti-bonding, whereas the higher energy bands at 322 and $326 \mathrm{~nm}$ are mainly of type $\mathrm{Co}(\mathrm{d})$ to $\mathrm{Co}(\mathrm{d})-\mathrm{H}(\mathrm{s}) \sigma$ anti-bonding.

For the ab initio calculations, the choice of an active space in the reference CASSCF is crucial to the success of the method. In this paper, we have decided to focus on smaller active spaces and leave discussion of larger spaces to related work that we have been doing on the spectra of cobalt-hydride complexes. ${ }^{60}$ These larger spaces include those that incorporate an additional set of correlating cobalt " $4 \mathrm{~d}$ " orbitals to account for the so-called "double-shell effect".

As an initial guess in this work, we assumed a $\mathrm{d}_{6}$ configuration for the cobalt, and started with the minimum active space of 6 electrons in 5 orbitals, which we denote CAS $(6,5)$. The resulting transition energies were then further refined with the NEVPT2 correction to correct for the lack of dynamic correlation. We found that state averaging among at least the lowest 10 singlet and 3 triplet roots was essential for obtaining good transition energies. In this case, there is a single lower energy band at $415 \mathrm{~nm}$ and two higher energy bands of comparable intensity at 339 and $341 \mathrm{~nm}$. Although the energies are reasonable, the intensity of the lower energy band is severely underestimated.

Increasing the size of the active space by the inclusion of two occupied and two virtual orbitals gives a CAS $(10,9)$ model. This greatly improves the intensities compared to the CAS(6,5) model, while maintaining the quality of the transition energies. The important orbitals involved in these transitions are shown in Figure 3. For convenience we label them as 
HOMO and LUMO even though all the orbitals in the active space can have partial occupancies. In this case, the HOMO has an occupancy of 1.53, with all the lower orbitals having an occupancy greater than this. By contrast the LUMO and LUMO+1 have 0.89 and 0.41 electrons, respectively, whereas the occupancies of the higher orbitals are much smaller $(\sim 0.01)$. The orbitals HOMO-2 to HOMO are predominantly of metal d-type, whereas the HOMO-3 and HOMO-4 are $\mathrm{Co}-\mathrm{H}$ and $\mathrm{Co}-\mathrm{N} \sigma$ bonding orbitals, and the LUMO and LUMO+1 are $\mathrm{Co}(\mathrm{d})-\mathrm{N}(\mathrm{p})$ and $\mathrm{Co}-\mathrm{H} \sigma$ anti-bonding orbitals, respectively. The CASSCF ground state is dominated $(>90 \%)$ by the reference, doubly-occupied configuration, [222220000]. The transitions at 415 and $421 \mathrm{~nm}$ are principally of metal d-orbital to LUMO $(\mathrm{Co}(\mathrm{d})-\mathrm{N}(\mathrm{p}) \sigma$ anti-bonding) character, whereas the higher energy bands at 313 and $317 \mathrm{~nm}$ are mostly of $\mathrm{Co}(\mathrm{d})$ to $\mathrm{Co}-\mathrm{H} \sigma$ anti-bonding type.

A comparison of the TDDFT and CASSCF/NEVPT2 results shows that they are of comparable quality and give very similar interpretations of the transitions. In this case, therefore, TDDFT is much more efficient than the ab initio method (even with relatively small active spaces), as it is much less computationally expensive, as well as being much more straightforward to apply.

\section{QMCF-MD Simulations}

This section discusses the simulation results. Equilibration in terms of system energy and temperature were achieved during the first 5 ps of the 25 ps simulations and so we have only used the last 20 ps of data in our analysis.

\section{Properties of the Complex}

A summary of the properties of some pertinent internal coordinates of the complexes during the simulation are provided in Table 2. The two molecules display similar behaviours with Table 2 significant variations of the internal coordinates around their average values. Perhaps the most marked difference between the complexes concerns the maximum and minimum values 
of the $\mathrm{Co}-\mathrm{N}$ bond lengths that are observed. Closer inspection indicates that these extremes are attained only once in the $\left[\mathrm{HCo}(\text { tmen })_{2}\right]^{+}$trajectories in events that last $100 \mathrm{fs}$ or less.

The dihedrals in the tables show that the central $\mathrm{CoN}_{4}$ cores of the complexes maintain planarity throughout the simulations although they have substantial flexibility. Plots of the $\mathrm{C}-\mathrm{N}-\mathrm{N}-\mathrm{C}$ dihedrals in the two complexes are illustrated in Figure 4. These indicate that Figure 4 there are two peaks for each complex that are centred around $\pm 45^{\circ}$, respectively. In the case of the methylated complex these peaks are separate with no exchange between them on the timescale of the simulation. However, the unmethylated complex flips conformations several times with quite significant periods spent with both dihedrals within the same peak.

The remaining heavy atom $\mathrm{C}-\mathrm{C}-\mathrm{C}-\mathrm{C}$ and $\mathrm{C}-\mathrm{C}-\mathrm{C}-\mathrm{N}$ dihedral degrees of freedom for the methylated complex have similar dynamics to their $\mathrm{C}-\mathrm{N}-\mathrm{N}-\mathrm{C}$ counterparts with oscillations within well-defined peaks but no exchange between different conformations. By contrast, the $\mathrm{C}-\mathrm{C}-\mathrm{C}-\mathrm{H}$ dihedral angles show very broad distributions within the range $\left[-180^{\circ}, 180^{\circ}\right]$, indicating that the methyl groups can readily rotate during the simulation.

Statistics for the partial charges of the central atoms in the complexes from the simulations are presented in Table 3. Charges are calculated with both the B3LYP and BP86 Table 3 functionals and using three different charge approximations, namely electrostatic potential fitting (ESP), Löwdin and Mulliken analyses. As might be expected the charges values vary quite widely, depending upon the analysis. The hydride has a small magnitude charge in all cases, although only the ESP method makes it negative. The cobalt atom is always positive and the nitrogen atoms are strongly negative except with the Löwdin method for which they are approximately neutral. Overall, the cobalt is less positive in the $\left[\mathrm{HCo}(\operatorname{tmen})_{2}\right]^{+}$complex and the nitrogens are more negative, whereas the B3LYP functional favors larger magnitudes for the cobalt and nitrogen charges than BP86. The decrease in partial charge of the cobalt upon methylation of the complex, irrespective of the employed functional and population analysis scheme, is consistent with the weak electron donor property of the methyl groups.

Power spectra of the complexes' vibrational modes were computed via Fourier transform 
of the atoms' velocity auto-correlation functions. The spectra are presented in Figure 5 and Figure 5 depict both common features and clear differences in the fine structure. The methylated species has more pronounced peaks overall, suggesting a more rigid structure and better defined modes. The Co-Hydride spectra of the methylated complex depicts a slight blueshift compared to the unmethylated species (bottom of the Figure). Experimentally, the Co-H stretching frequency has been reported to be in the range $1930-1970 \mathrm{~cm}^{-1}$ by Saillant et $a l .{ }^{61}$ although for complexes containing phosphine ligands as stabilizers. The relatively high $\mathrm{Co}-\mathrm{H}$ stretching frequency in the present case could be attributable to the absence of such axial ligands.

To investigate how the electronic transitions of the complexes change along the QMCFMD simulations, we performed TDDFT calculations on 500 equally-spaced snapshots taken from the $\left[\mathrm{HCo}(\text { tmen })_{2}\right]^{+}$simulation, using the B3LYP functional and both the 6-31G(d,p) and TZVP basis sets. The results are plotted as histograms in Figure 6 and are to be compared with the static B3LYP spectra calculated at the optimized geometries in Table 1. In both histograms, two peaks can be discerned which correspond to those of the two principal bands in the static spectra. These occur at slightly longer wavelengths in the dynamic spectra than in their static counterparts with values at approximately 360 and $340 \mathrm{~nm}$ and at 470 and $450 \mathrm{~nm}$ with the 6-31G(d,p) and TZVP basis sets, respectively. The major difference, however, in the dynamic spectra is that the shorter wavelength bands are now dominant, whereas the longer wavelength bands are almost washed out with amplitudes that are only slightly above the background.

\section{Solvation Structure}

As initial analyses we examined the spherical radial distribution functions (RDFs) of the cobalt and hydride with the water molecules. The cobalt-water oxygen RDFs are shown in Figure 7 , from which it can be seen that the solvation around $\left[\mathrm{HCo}(\operatorname{tmen})_{2}\right]^{+}$has a more Figure 7 pronounced shell structure than $\left[\mathrm{HCo}(\mathrm{en})_{2}\right]^{+}$. The figure also shows the distribution of 
the smallest $\mathrm{Co}-\mathrm{O}$ (water) distances that are observed in the MD simulations. The most likely distance for both complexes is approximately $2.2 \AA$, which is what is observed in a structure that is geometry optimized with water as a coordinating ligand. For $\left[\mathrm{HCo}(\mathrm{en})_{2}\right]^{+}$ the distribution drops off sharply after this although there is still a substantial fraction of the population $(\sim 8 \%)$ that has longer $\mathrm{Co}-\mathrm{O}$ coordination distances than $3.0 \AA$. By constrast, the $\left[\mathrm{HCo}(\text { tmen })_{2}\right]^{+}$distribution is clearly bimodal with a first peak containing about $65 \%$ of the population up to about $2.9 \AA$, and a second peak with the remainder of the structures with a maximum at $3.3 \AA$. The different distributions of the shortest Co-O distance show that the water-coordination site of the methylated complex is not populated in a rather larger number of configurations. This is not the case in the unmethylated system, where the coordination site is predominantly occupied by a water molecule. This clearly shows the important steric effects that the methyl groups have on water coordination.

The RDFs for the hydride-water oxygen distances are shown in Figure 8. These depict a Figure 8 decreased intensity of hydration in the vicinity of the Co center, within $\sim 6 \AA$. The relative peak intensities indicate that the hydride is more exposed to the solvent in the unmethylated complex, whereas the methylated complex shows a clear peak at about $8 \AA$, which is associated with the oxygens that interact with the methyl groups.

SLICE projections of the complexes are given in Figure 9. Each shows three distributions: Figure 9 the water-oxygen distribution around the complexes in green and blue, the complexes in gray, and the hydride explicitly in red. The molecular plane employed for the projection was determined from the four nitrogen atoms and the spacing of the projection planes was $1.5 \AA$. Starting at the central panels that represent the molecular plane, panels ' +1 ' to ' +4 ' show the hydration above the plane, and panels '-1' to '-4' the hydration below the plane.

The most striking difference between the two complexes is the much more highly organized solvation structure for $\left[\mathrm{HCo}(\operatorname{tmen})_{2}\right]^{+}$with well-defined solvation hot-spots occurring in all planes, except ' +4 '. By contrast, the only major solvation peaks for the unmethylated $\left[\mathrm{HCo}(\mathrm{en})_{2}\right]^{+}$occur in the ' +1 ' and, to a lesser extent, the '-1' planes in positions that bridge 
the two terminal amine groups of the $\left[\mathrm{HCo}(\mathrm{en})_{2}\right]^{+}$ligands. These results emphasis the significant ordering effects that methyl groups can have on solvent structure. Concerning the complexes themselves, the major difference is that the hydride distribution is much broader in the unmethylated case, indicating a higher degree of flexibility when the hydride is not encaged by the complex. This agrees with the results of the spherical radial distribution analysis.

Hydration layer statistics from the Laguerre tesselation analysis is presented in Table 4 Table 4 and the corresponding TCFs in Figure 10. As might be expected the hydration layers are Figure 10 larger for the methylated complex. The number of exchanges and residence times indicate that the inner solvation layers of $\left[\mathrm{HCo}(\mathrm{tmen})_{2}\right]^{+}$are more structured than those of $\left[\mathrm{HCo}(\mathrm{en})_{2}\right]^{+}$although this difference is less notable in the outer layer. Differences between the complexes are clearer in a comparison of the TCFs, $C(s)$ and $S(s)$, which decay much more quickly for $\left[\mathrm{HCo}(\mathrm{en})_{2}\right]^{+}$than $\left[\mathrm{HCo}(\mathrm{tmen})_{2}\right]^{+}$. The short-time correlation times, $\tau_{s}$, from the double exponential fit are only slightly greater for $\left[\mathrm{HCo}(\mathrm{tmen})_{2}\right]^{+}$in each of the layers, whereas the long-time contributions, $\tau_{l}$, are between $50 \%$ and $100 \%$ greater. This indicates that the minimum residence times are similar for both simulations, whereas the maximum residence times clearly differ with the methylated species featuring a higher degree of structure in the bulk region, and $\left[\mathrm{HCo}(\mathrm{en})_{2}\right]^{+}$having a structure that is closer to that of bulk water. ${ }^{57,58}$

As a final illustration of the data that is provided by Laguerre tessellation, some molecular and atomic volumes and surfaces for the complexes are presented in Table 5. The effective Table 5 surface is the surface that an atom or a molecule shares with the surrounding water, and provides an estimate of the solvent accessible surface. Not surprisingly the volumes and areas obtained by this analysis are larger for the methylated species, although this is not reflected by the atomic volume and surface of the hydride as only minor decreases in these two properties are observed. 


\section{Conclusions}

In this study we have simulated two tetramine $\mathrm{Co}(\mathrm{III})$-hydrido compounds that were chosen as models of the intermediates that are known to be important in the hydrogen-evolving catalytic cycles of the cobaloxime complexes. We used both static DFT and hybrid potential molecular dynamical QMCF-MD techniques and compared our results to the available experimental structural and spectral data. A major goal of this work has been to provide a foundation for future simulations of hydrogen-evolving catalysts that we intend to carry out using adaptive QC/MM molecular dynamics methods of the QMCF-MD type. Such dynamical techniques appear to us to be essential for obtaining a full and accurate understanding of the behaviours and properties of these complexes in their functional environments.

\section{Acknowledgement}

Financial support for AB, MJF and VA from the French National Research Agency (NiFeCat, ANR-10-BLAN-7-11), the FP7 CEA-Eurotalent COFUND Programme, and the CEA's DSV-ENERGIE 2011 Programme is gratefully acknowledged. AKHW thanks the LeopoldFranzens-University of Innsbruck (Rector Prof. Dr. Dr.hc. Tilmann Märk) for a PhD scholarship. AKHW and THH acknowledge the support by the Austrian Ministry of Science BMWF UniInfrastrukturprogramm as part of the Research Platform Scientific Computing at the University of Innsbruck. The COST Action CM1202 PERSPECT-H2O is also acknowledged.

\section{References}

(1) Artero, V.; Chavarot-Kerlidou, M.; Fontecave, M. Splitting Water with Cobalt. Angew. Chem. Int. Ed. 2011, 50, $7238-7266$.

(2) Dempsey, J.; Brunschwig, B.; Winkler, J.; Gray, H. Hydrogen Evolution Catalyzed by Cobaloximes. Acc. Chem. Res. 2009, 42, 1995-2004. 
(3) Chao, T.; Espenson, J. Mechanism of Hydrogen Evolution from Hydridocobaloxime. J. Am. Chem. Soc. 1978, 100, 129-133.

(4) Jacques, P.; Artero, V.; Pécaut, J.; Fontecave, M. Cobalt and Nickel Diimine-dioxime Complexes as Molecular Electrocatalysts for Hydrogen Evolution with Low Overvoltages. Proc. Natl. Acad. Sci. 2009, 106, 20627-20632.

(5) Dempsey, J.; Winkler, J.; Gray, H. Mechanism of $\mathrm{H}_{2}$ Evolution from a Photogenerated Hydridocobaloxime. J. Am. Chem. Soc. 2010, 132, 16774-16776.

(6) Razavet, M.; Artero, V.; Fontecave, M. Proton Electroreduction Catalyzed by Cobaloximes: Functional Models for Hydrogenases. Inorg. Chem. 2005, 44, 4786-4795.

(7) Hu, X.; Cossairt, B.; Brunschwig, B.; Lewis, N.; Peters, J. Electrocatalytic Hydrogen Evolution by Cobalt Difluoroboryl-diglyoximate Complexes. Chem. Commun. 2005, $4723-4725$.

(8) Baffert, C.; Artero, V.; Fontecave, M. Cobaloximes as Functional Models for Hydrogenases. 2. Proton Electroreduction Catalyzed by Difluoroborylbis (Dimethylglyoximato) Cobalt (II) Complexes in Organic Media. Inorg. Chem. 2007, 46, 1817-1824.

(9) Hu, X.; Brunschwig, B.; Peters, J. Electrocatalytic Hydrogen Evolution at Low Overpotentials by Cobalt Macrocyclic Glyoxime and Tetraimine Complexes. J. Am. Chem. Soc. 2007, 129, 8988-8998.

(10) Pantani, O.; Anxolabehere-Mallart, E.; Aukauloo, A.; Millet, P. Electroactivity of Cobalt and Nickel Glyoximes with regard to the Electro-reduction of Protons into Molecular Hydrogen in Acidic Media. Electrochem. Commun. 2007, 9, 54-58.

(11) Dempsey, J.; Winkler, J.; Gray, H. Kinetics of Electron Transfer Reactions of $\mathrm{H}_{2}{ }^{-}$ Evolving Cobalt Diglyoxime Catalysts. J. Am. Chem. Soc. 2009, 132, 1060-1065. 
(12) Du, P.; Schneider, J.; Luo, G.; Brennessel, W.; Eisenberg, R. Visible Light-Driven Hydrogen Production from Aqueous Protons Catalyzed by Molecular Cobaloxime Catalysts. Inorg. Chem. 2009, 48, 4952-4962.

(13) Du, P.; Knowles, K.; Eisenberg, R. A Homogeneous System for the Photogeneration of Hydrogen from Water based on a Platinum (II) Terpyridyl Acetylide Chromophore and a Molecular Cobalt Catalyst. J. Am. Chem. Soc. 2008, 130, 12576-12577.

(14) Lazarides, T.; McCormick, T.; Du, P.; Luo, G.; Lindley, B.; Eisenberg, R. Making Hydrogen from Water using a Homogeneous System without Noble Metals. J. Am. Chem. Soc. 2009, 131, 9192-9194.

(15) McCormick, T.; Calitree, B.; Orchard, A.; Kraut, N.; Bright, F.; Detty, M.; Eisenberg, R. Reductive Side of Water Splitting in Artificial Photosynthesis: New Homogeneous Photosystems of Great Activity and Mechanistic Insight. J. Am. Chem. Soc. 2010, 132, 15480-15483.

(16) Muckerman, J.; Fujita, E. Theoretical Studies of the Mechanism of Catalytic Hydrogen Production by a Cobaloxime. Chem. Commun. 2011, 47, 12456-12458.

(17) Solis, B.; Hammes-Schiffer, S. Theoretical Analysis of Mechanistic Pathways for Hydrogen Evolution Catalyzed by Cobaloximes. Inorg. Chem. 2011, 50, 11252-11262.

(18) Bhattacharjee, A.; Andreiadis, E. S.; Chavarot-Kerlidou, M.; Fontecave, M.; Field, M. J.; Artero, V. A Computational Study of the Mechanism of Hydrogen Evolution by Cobalt(Diimine-Dioxime) Catalysts. Chem. Europ. J. 2013, 19, 15166-15174.

(19) Bhattacharjee, A.; Chavarot-Kerlidou, M.; Andreiadis, E. S.; Fontecave, M.; Field, M. J.; Artero, V. Combined Experimental and Theoretical Characterization of the Hydrido-Cobaloxime [HCo(dmgH)2(PnBu3)]. Inorg. Chem. 2012, 51, 7087-7093. 
(20) Schrauzer, G.; Holland, R. Hydridocobaloximes. J. Am. Chem. Soc. 1971, 93, 15051506.

(21) Rahman, A.; Jackson, W.; Willis, A.; Rae, A. Synthesis and Crystal and Molecular Structure of a Hydrido Tetraamine Cobalt (III) Complex. Chem. Commun. 2003, 27482749 .

(22) Neese, F. The ORCA Program System. WIREs Comput Mol Sci 2012, 2, 73-78.

(23) Becke, A. Density-Functional Thermochemistry. III. The Role of Exact Exchange. Chem. Phys. 1993, 98, 5648-5652.

(24) Perdew, J. Density-Functional Approximation for the Correlation Energy of the Inhomogeneous Electron Gas. Phys. Rev. B 1986, 33, 8822-8824.

(25) Becke, A. Density-Functional Exchange-Energy Approximation with Correct Asymptotic Behavior. Phys. Rev. A 1988, 38, 3098-3100.

(26) Lee, C.; Yang, W.; Parr, R. Development of the Colle-Salvetti Correlation-Energy Formula into a Functional of the Electron Density. Phys. Rev. B 1988, 37, 785-789.

(27) Weigend, F.; Ahlrichs, R. Balanced Basis Sets of Split Valence, Triple Zeta Valence and Quadruple Zeta Valence Quality for H to Rn: Design and Assessment of Accuracy. Phys. Chem. Chem. Phys. 2005, 7, 3297-3305.

(28) Kornobis, K.; Kumar, N.; Wong, B.; Lodowski, P.; Jaworska, M.; Andruniów, T.; Ruud, K.; Kozlowski, P. Electronically Excited States of Vitamin B 12 $_{\text {: Benchmark }}$ Calculations Including Time-Dependent Density Functional Theory and Correlated ab Initio Methods. J. Phys. Chem. A 2011, 115, 1280-1292.

(29) Andruniów, T.; Jaworska, M.; Lodowski, P.; Zgierski, M.; Dreos, R.; Randaccio, L.; Kozlowski, P. Time-Dependent Density Functional Theory Study of Cobalt Corrinoids: Electronically Excited States of Coenzyme B 12 . J. Chem. Phys. 2009, 131, 105105. 
(30) Kozlowski, P.; Kumar, M.; Piecuch, P.; Li, W.; Bauman, N.; Hansen, J.; Lodowski, P.; Jaworska, M. The Cobalt-Methyl Bond Dissociation in Methylcobalamin: New Benchmark Analysis Based on Density Functional Theory and Completely Renormalized Coupled-Cluster Calculations. 2012, 8, 1870-1894.

(31) Kornobis, K.; Kumar, N.; Lodowski, P.; Jaworska, M.; Piecuch, P.; Lutz, J.; B.M., W.; Kozlowski, P. Electronic Structure of the S1 State in Methylcobalamin: Insight from CASSCF/MC-XQDPT2, EOM-CCSD, and TD-DFT Calculations. J. Comput. Chem. 2013, 113, 479-488.

(32) Sinnecker, S.; Rajendran, A.; Klamt, A.; Diedenhofen, M.; Neese, F. Calculation of Solvent Shifts on Electronic G-Tensors with the Conductor-Like Screening Model (COSMO) and its Self-Consistent Generalization to Real Solvents (COSMO-RS). J. Phys. Chem. A 2006, 110, 2235-2245.

(33) Angeli, C.; Cimiraglia, R.; Evangelisti, S.; Leininger, T.; Malrieu, J. Introduction of NElectron Valence States for Multireference Perturbation Theory. J. Chem. Phys. 2001, $114,10252-10264$.

(34) Andersson, K.; Malmqvist, P.; Roos, B. Second-Order Perturbation Theory with a Complete Active Space Self-Consistent Field Reference Function. J. Chem. Phys. 1992, 96, 1218-1226.

(35) Hofer, T. S.; Pribil, A. B.; Randolf, B. R.; Rode, B. M. Ab Initio Quantum Mechanical Charge Field Molecular Dynamics - A Nonparametrized First-Principle Approach to Liquids and Solutions. Advances in Quantum Chemistry 2010, 59, 213-246.

(36) Hofer, T. S.; Rode, B. M.; Pribil, A. B.; Randolf, B. R. Simulations of Liquids and Solutions Based on Quantum Mechanical Forces. Adv. Inorg. Chem. 2010, 62, 143175 . 
(37) Rode, B. M.; Hofer, T. S.; Randolf, B. R.; Schwenk, C. F.; Xenides, D.; Vchirawongkwin, V. Ab Initio Quantum Mechanical Charge Field (QMCF) Molecular Dynamics: a QM/MM-MD Procedure for Accurate Simulations of Ions and Complexes. Theor.Chem.Acc. 2006, 115, 77-85.

(38) Hofer, T. S.; Rode, B. M. How to Access Structure and Dynamics of Solutions: The Capabilities of Computational Methods. Pure Appl.Chem. 2006, 78, 525-539.

(39) Weiss, A. K.; Hofer, T. S. Exploiting the Capabilities of Quantum Chemical Simulations to Characterise the Hydration of Molecular Compounds. RSC Adv. 2013, 3, 1606-1635.

(40) Mulliken, R. S. Electronic Population Analysis on LCAO-MO Molecular Wave Functions. I. J. Chem. Phys. 1955, 23, 1833-1840.

(41) Vchirawongkwin, V.; Persson, I.; Rode, B. M. Structure and Dynamics of Sulfate Ion in Aqueous Solution: An ab initio QMCF MD Simulation and Large Angle X-ray Scattering Study. J.Phys.Chem. B 2007, 111, 4150-4155.

(42) Pribil, A. B.; Hofer, T. S.; Vchirawongkwin, V.; Randolf, B. R.; Rode, B. M. Quantum Mechanical Simulation Studies of Molecular Vibrations and Dynamics of Oxo-Anions in Water. Chem.Phys. 2008, 346, 182-185.

(43) Schwenk, C. F.; Hofer, T. S.; Randolf, B. R.; Rode, B. M. The Influence of Heteroligands on the Reactivity of $\mathrm{Ni}^{2+}$ in Solution. Phys.Chem.Chem.Phys. 2005, 7, 1669-1673.

(44) Hofer, T. S.; Randolf, B. R.; Rode, B. M. The Influence of Quantum Forces on Molecular Dynamics Simulation Results for Hydrated Aluminium (III). Chem.Phys.Lett. 2006, 422, 492-495.

(45) Allen, M. P.; Tildesley, D. J. Computer Simulation of Liquids; Oxford Science Publications: Oxford, 1990. 
(46) Wang, J.; Wolf, R. M.; Caldwell, J. W.; Kollman, P. A.; Case, D. A. Development and Testing of a General Amber Force Field. J. Comput. Chem. 2004, 25, 1157-1174.

(47) Liew, C. C.; Inomata, H.; Arai, K. Flexible Molecular Models for Molecular Dynamics Study of Near and Supercritical Water. Fluid Phase Equilibr. 1998, 144, 287-298.

(48) Onsager, L. Electric Moments of Molecules in Liquids. J.Am.Chem.Soc. 1936, 58, 14861493.

(49) Berendsen, H. J. C.; Postma, J. P. M.; van Gusteren, W. F.; Nola, A. D.; Haak, J. R. Molecular Dynamics with Coupling to an External Bath. J.Chem.Phys. 1984, 81, 36843690.

(50) TURBOMOLE V6.4 2012, a development of University of Karlsruhe and Forschungszentrum Karlsruhe GmbH, 1989-2007, TURBOMOLE GmbH, since 2007; available from http://www.turbomole.com.

(51) Weiss, A. K. H.; Hofer, T. S.; Randolf, B. R.; Bhattacharjee, A.; Rode, B. M. Hydrogen Bond Formation of Formamide and N-Methylformamide in Aqueous Solution Studied by Quantum Mechanical Charge Field-Molecular Dynamics (QMCF-MD). Phys.Chem.Chem.Phys. 2011, 13, 12173-12185.

(52) Rycroft, C. H. VORO++: A Three-Dimensional Voronoi Cell Library in C++. Chaos: An Interdisciplinary Journal of Nonlinear Science 2009, 19, 04111.

(53) Poupon, A. Voronoi and Voronoi-Related Tesselations in Studies of Protein Structure and Interaction. Current Opinion in Structural Biology 2004, 14, 233-241.

(54) Voronoi, G. Nouvelles Applications des Paramétres Continus à la Théorie des Formes Quadratiques. Journal für die Reine und Angewandte Mathematik 1908, 133, 97-178.

(55) Bondi, A. Van der Waals Volumes and Radii. J. Phys. Chem. 1964, 68, 441-451. 
(56) Hofer, T. S.; Tran, H. T.; Schwenk, C. F.; Rode, B. M. Characterisation of Dynamics and Reactivities of Solvated Ions by Ab Initio Simulations. J. Comput. Chem. 2004, 25, 211-214.

(57) Xenides, D.; Randolf, B. R.; Rode, B. M. Structure and Ultrafast Dynamics of Liquid Water: A Quantum Mechanics/Molecular Mechanics Molecular Dynamics Simulation Study. J.Chem.Phys. 2005, 122, 174506.

(58) Coudert, F.-X.; Vuilleumier, R.; Boutin, A. Dipole Moment, Hydrogen Bonding and IR Spectrum of Confined Water. Chem.Phys.Chem. 2006, 7, 2464-2467.

(59) Chanda, J.; Chakraborty, S.; Bandyopadhyay, S. Sensitivity of Hydrogen Bond Lifetime Dynamics to the Presence of Ethanol at the Interface of a Phospholipid Bilayer. J.Phys.Chem.B 2006, 110, 3791-3797.

(60) A. Bhattarcharjee et al., to be submitted.

(61) Kaesz, H.; Saillant, R. Hydride Complexes of the Transition Metals. Chem. Rev. 1972, 72, 231-281. 
Table 1: Comparison of experimental and theoretical UV-VIS absorption spectra for $\left[\mathrm{HCo}(\text { tmen })_{2}\right]^{+}$. Relative intensities with respect to the strongest transition are in parentheses after the wavelength values.

\begin{tabular}{lll}
\hline \hline Method & $\lambda_{1} / \mathrm{nm}$ & $\lambda_{2} / \mathrm{nm}$ \\
\hline \hline & $444(1.00)$ & $340(0.53)$ \\
Experiment & & \\
DFT & & \\
\hline & & \\
BP86/6-31G(d,p) & $430(1.00), 436(0.10)$ & $354(0.35)$ \\
BP86/TZVP & $401(0.65), 398(1.00)$ & $334(0.10), 337(0.10)$ \\
B3LYP/6-31G(d,p) & $439(1.00), 442(0.25)$ & $336(0.75)$ \\
B3LYP/TZVP & $418(0.25), 415(1.00)$ & $326(0.50), 322(0.03)$ \\
CAS/NEVPT2 & & \\
\hline & & $341(1.00), 339(1.00)$ \\
CAS(6,5)/NEVPT2 & $415(<0.01)$ & \\
CAS(10,9)/NEVPT2 & $422(0.40), 415(0.40)$ & $317(1.00), 313(0.70)$ \\
& & \\
\hline
\end{tabular}


Table 2: Table of various internal coordinates calculated from $20 \mathrm{ps}$ of simulation. Distances are in $\AA$, and angles and dihedrals in degrees. The values in the table are computed for all possible internal coordinates of the same type.

\begin{tabular}{l|rllrr|rrrrr}
\hline \hline & \multicolumn{4}{|c}{$\left[\mathrm{HCo}(\mathrm{en})_{2}\right]^{+}$} & \multicolumn{5}{c}{$\left[\mathrm{HCo}(\mathrm{tmen})_{2}\right]^{+}$} \\
Internal Coordinate & \multicolumn{1}{|c}{ Avrg. \& Std. Dev. } & Max. & Min. & Avrg. \& Std. Dev. & Max. & Min. \\
\hline \hline Co-H (hyd.) & 1.42 & \pm & 0.09 & 1.84 & 1.15 & 1.41 & \pm & 0.09 & 1.79 & 1.15 \\
Co-N & 2.04 & \pm & 0.12 & 2.62 & 1.71 & 2.04 & \pm & 0.11 & 2.87 & 1.62 \\
N-Co-H (hyd.) & 89.8 & \pm & 9.2 & 140.3 & 54.5 & 90.7 & \pm & 10.3 & 141.6 & 54.7 \\
Co-N-H (amine) & 110.0 & \pm & 10.3 & 155.6 & 60.6 & 109.4 & \pm & 11.1 & 160.9 & 61.0 \\
Co-N-N-N & 0.4 & \pm & 6.3 & 22.6 & -33.0 & -1.0 & \pm & 6.1 & 24.1 & -23.6 \\
N-N-N-N & -0.1 & \pm & 8.6 & 28.8 & -32.7 & 0.1 & \pm & 7.8 & 30.6 & -24.4 \\
\hline \hline
\end{tabular}


Table 3: Table of the means and standard deviations of partial charges for atoms in the complexes, computed from 20 ps of simulation. Charges are calculated with the TZVP basis set, with both the B3LYP and BP86 functionals, and using the ESP, Löwdin and Mulliken approximations.

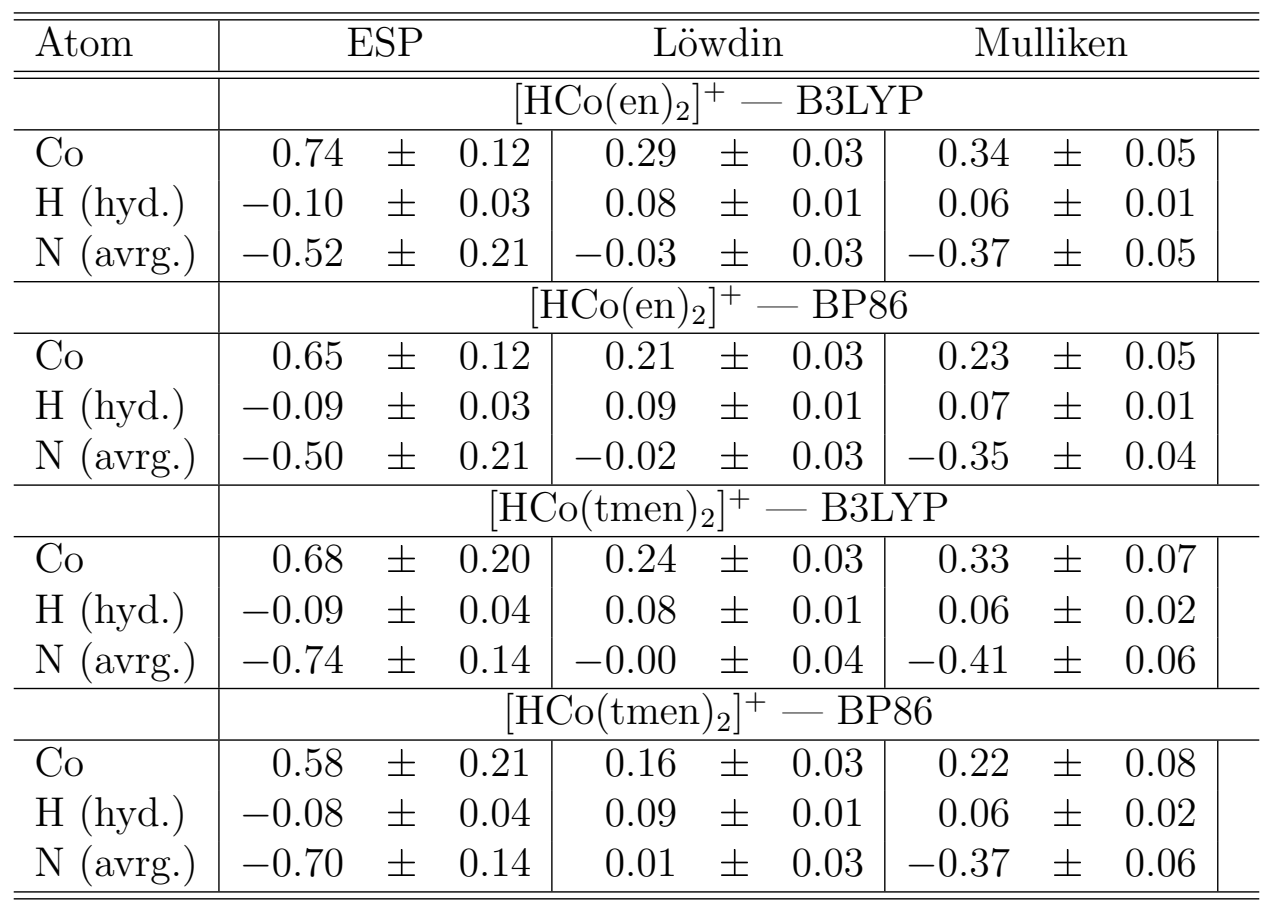


Table 4: Top: table of coordination numbers (CND), exchanges, mean residence times (MRT) and the ration of exchanges $\left(R_{\mathrm{ex}}\right)$ of water molecules within distinct hydration layers, obtained via Laguerre tessellation. The exchange data of the $i$ th layer refers to events with the $i+1$ th layer. This data is based on 20 ps of sampling. Bottom: table of computed $C(t)$ and $S(t)$ correlation times for the residence time of water molecules within distinct hydration layers, obtained via Laguerre tessellation. This data is based on 20 ps of sampling. All times are in ps.

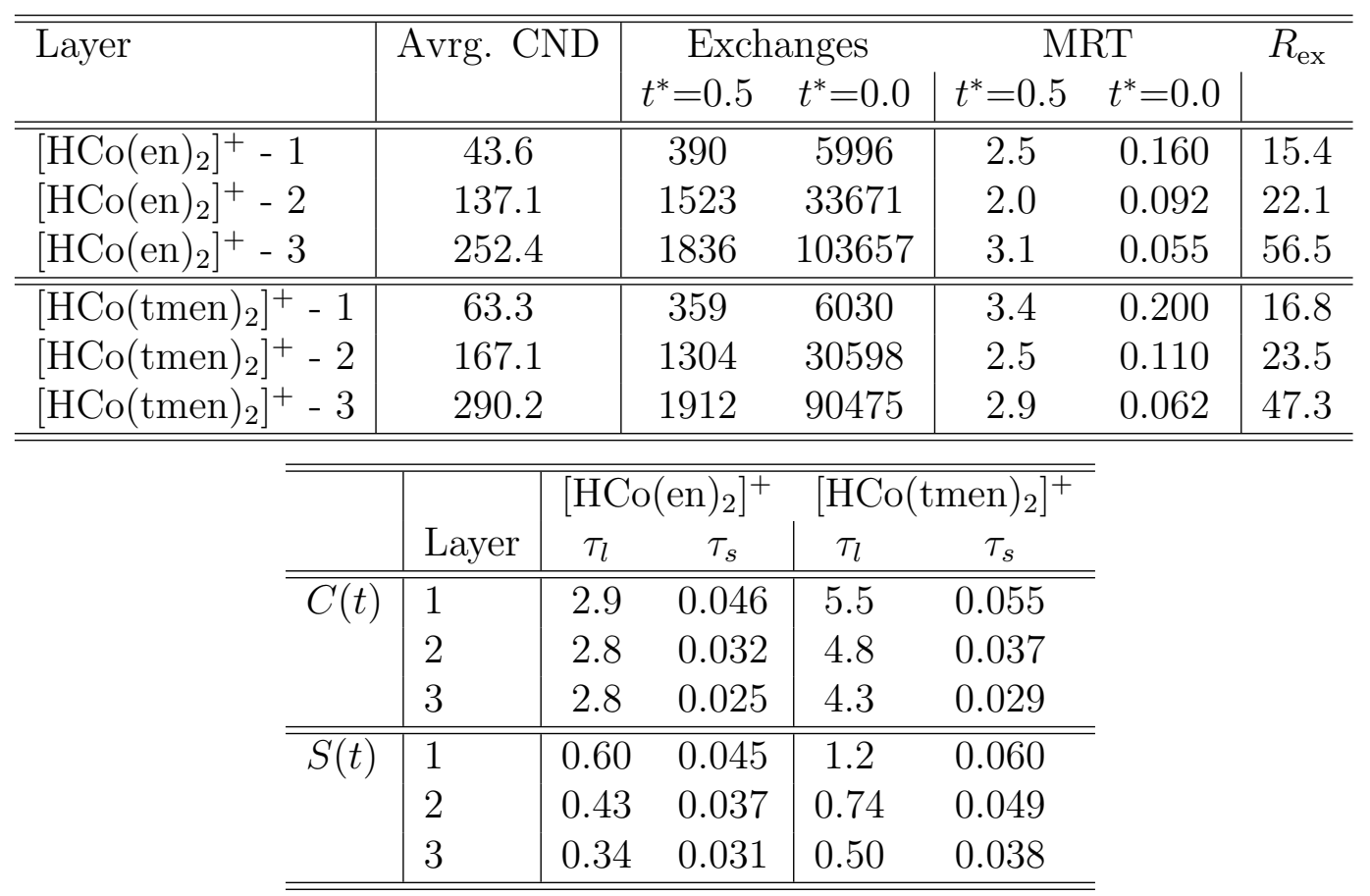


Table 5: Table of atomic and molecular volumes $(V)$ and surfaces $(A)$ obtained via Laguerre tessellation for the hydride atom and the complexes. Data is based on 20 ps of sampling and the averages and standard deviations for each property are shown.

\begin{tabular}{l|ccc|ccc|ccc|ccc|}
\hline \hline Complex & \multicolumn{3}{|c}{$V_{\text {Hydride }} \AA^{3}$} & \multicolumn{3}{c}{$A_{\text {Hydride }} \AA^{2}$} & \multicolumn{2}{c}{$V_{\text {complex }} \AA^{3}$} & \multicolumn{3}{c}{$A_{\text {complex }} \AA^{2}$} \\
\hline \hline$\left[\mathrm{HCo}(\mathrm{en})_{2}\right]^{+}$ & 13.6 & \pm & 3.4 & 31.9 & \pm & 5.0 & 318.3 & \pm & 24.8 & 320.5 & \pm & 18.2 \\
{$\left[\mathrm{HCo}(\mathrm{tmen})_{2}\right]^{+}$} & 12.0 & \pm & 3.1 & 29.8 & \pm & 4.8 & 559.1 & \pm & 26.8 & 461.7 & \pm & 15.9 \\
\hline \hline
\end{tabular}



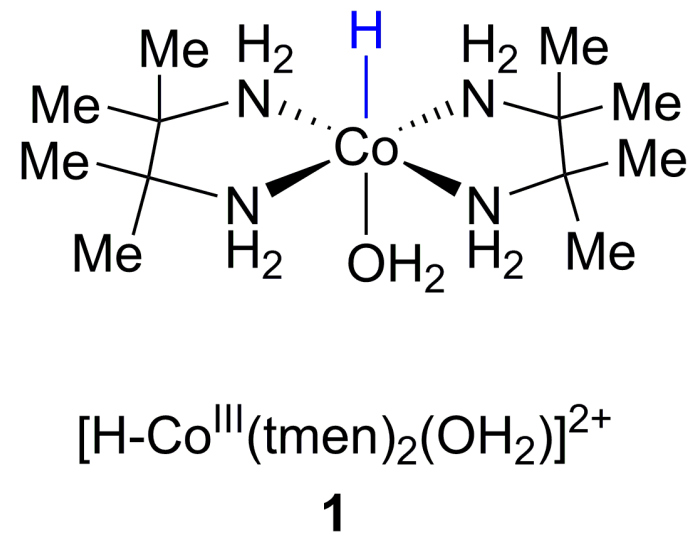
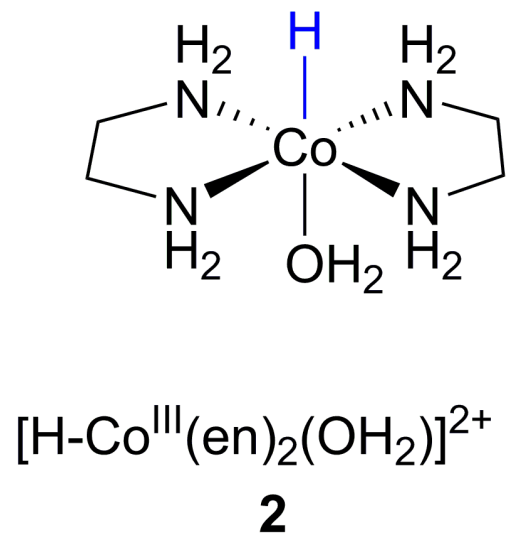

Figure 1: The hydrido-tetramine-cobalt(III) complexes studied in this work. Left: $\left[\mathrm{HCo}(\text { tmen })_{2}\right]^{+}$; right: $\left[\mathrm{HCo}(\mathrm{en})_{2}\right]^{+}$. 

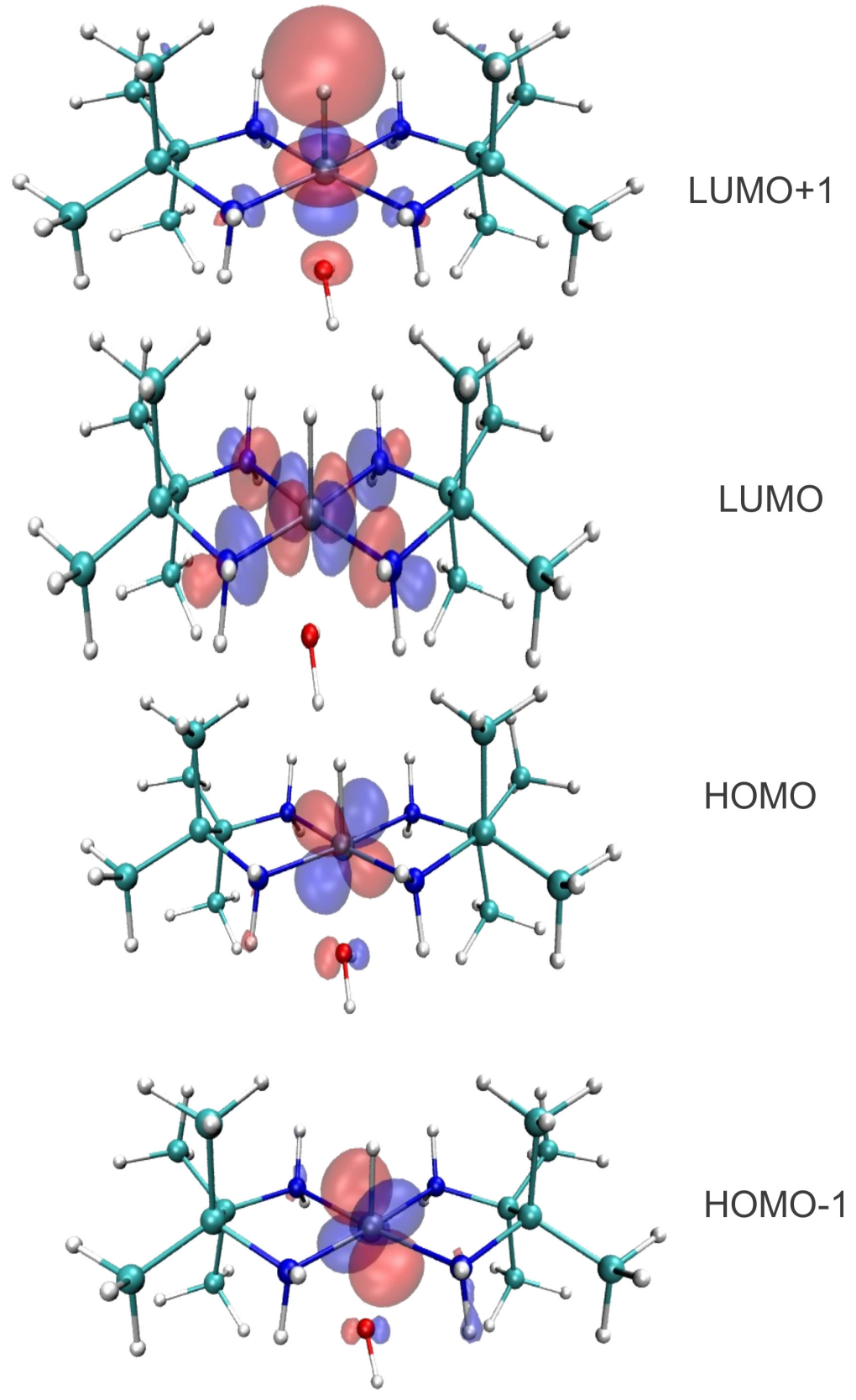

Figure 2: Isosurface plots of the Kohn-Sham orbitals from the B3LYP/TZVP DFT calculations that are important in the UV-VIS absorption spectrum transitions. 

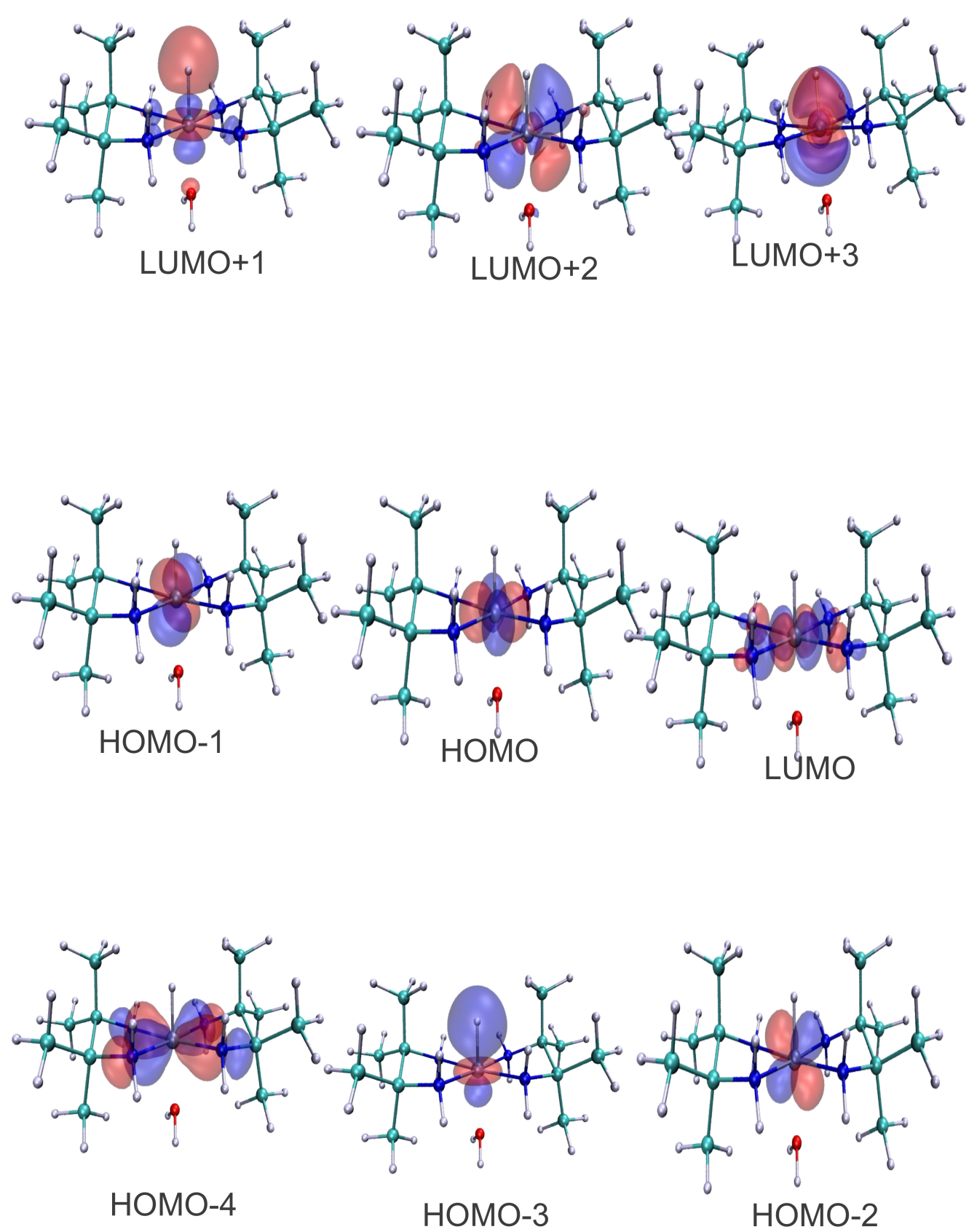

Figure 3: Isosurface plots of the orbitals from the CAS(10,9) CASSCF calculations that are important in the UV-VIS absorption spectrum transitions. 

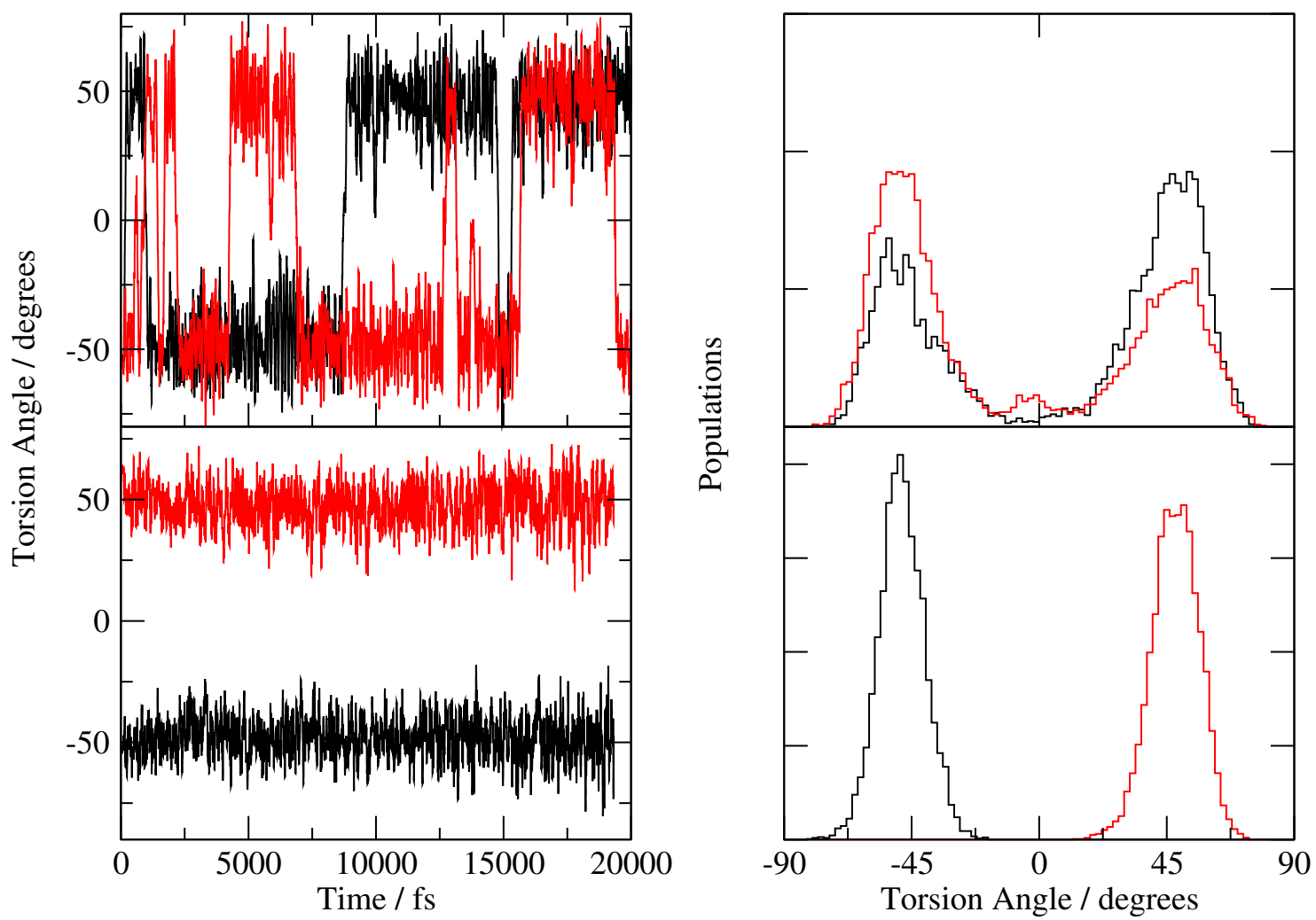

Figure 4: Trajectories (left) and histogram representations (right) of the $\mathrm{N}-\mathrm{C}-\mathrm{C}-\mathrm{N}$ dihedral angles. Top: $\left[\mathrm{HCo}(\mathrm{en})_{2}\right]^{+}$; bottom: $\left[\mathrm{HCo}(\mathrm{tmen})_{2}\right]^{+}$. 

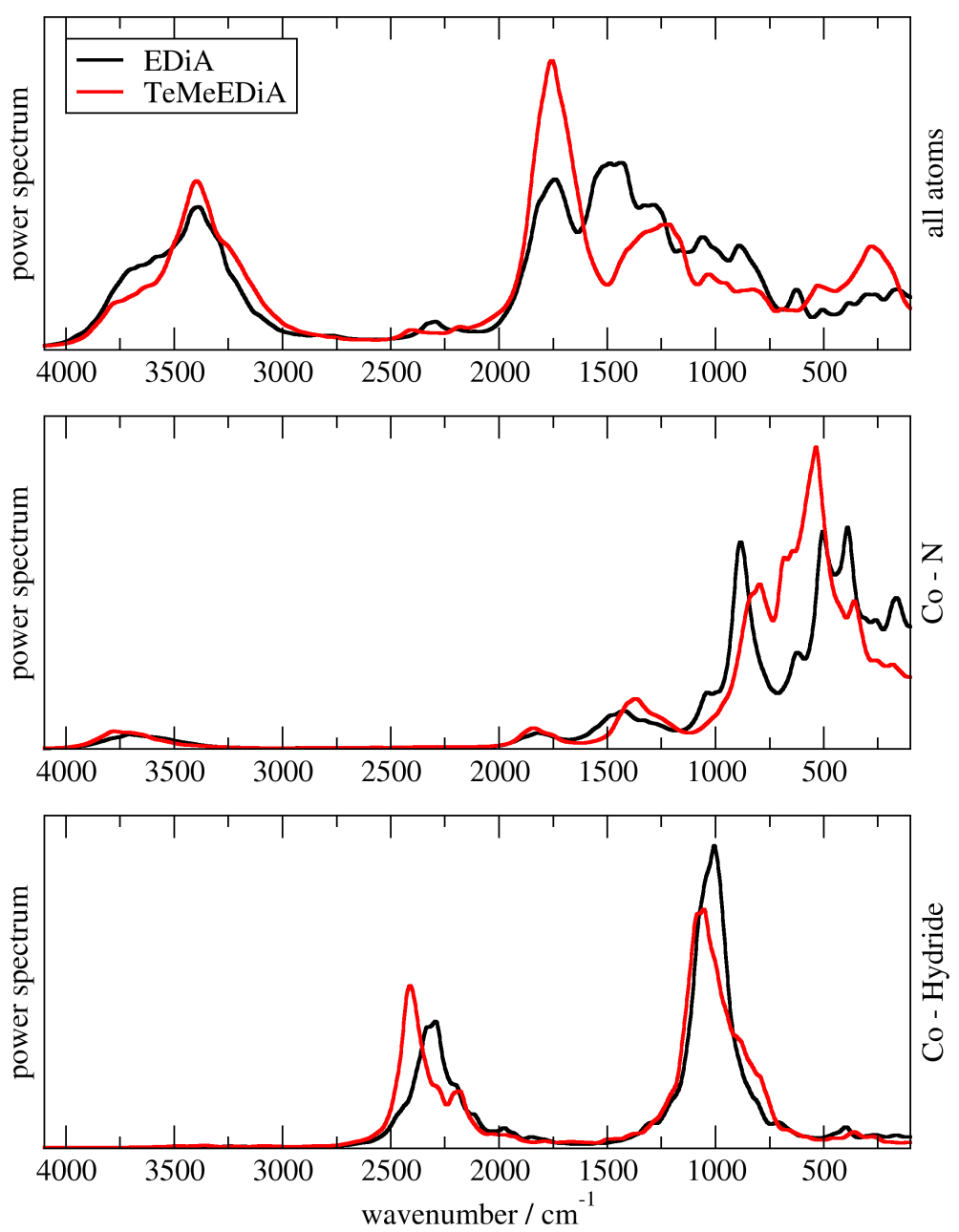

Figure 5: Power spectra of the complexes' vibrational modes, obtained via Fourier transform of the relevant velocity auto-correlation functions. Top: all atoms of the complexes; middle: Co-N bonds; bottom: Co-H (hyd.) bond. 


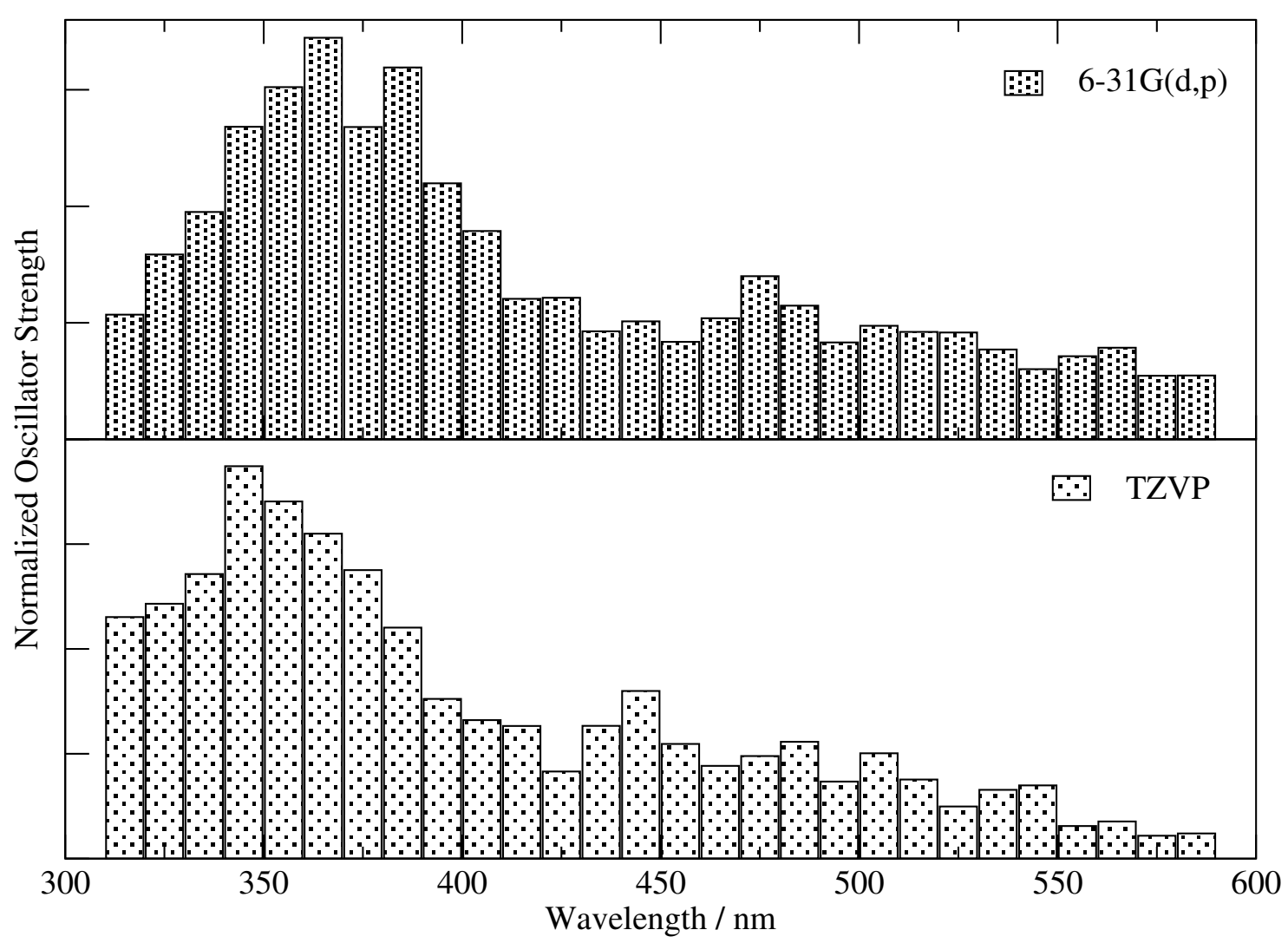

Figure 6: A histogram of the time-averaged wavelengths of the electronic transitions for the $\left[\mathrm{HCo}(\text { tmen })_{2}\right]^{+}$complex calculated at the TDDFT level of theory using the B3LYP functional and the 6-31G(d,p) (top) and TZVP (bottom) basis sets. The intensities are the normalized oscillator strengths and the histogram bin width is $10 \mathrm{~nm}$. 

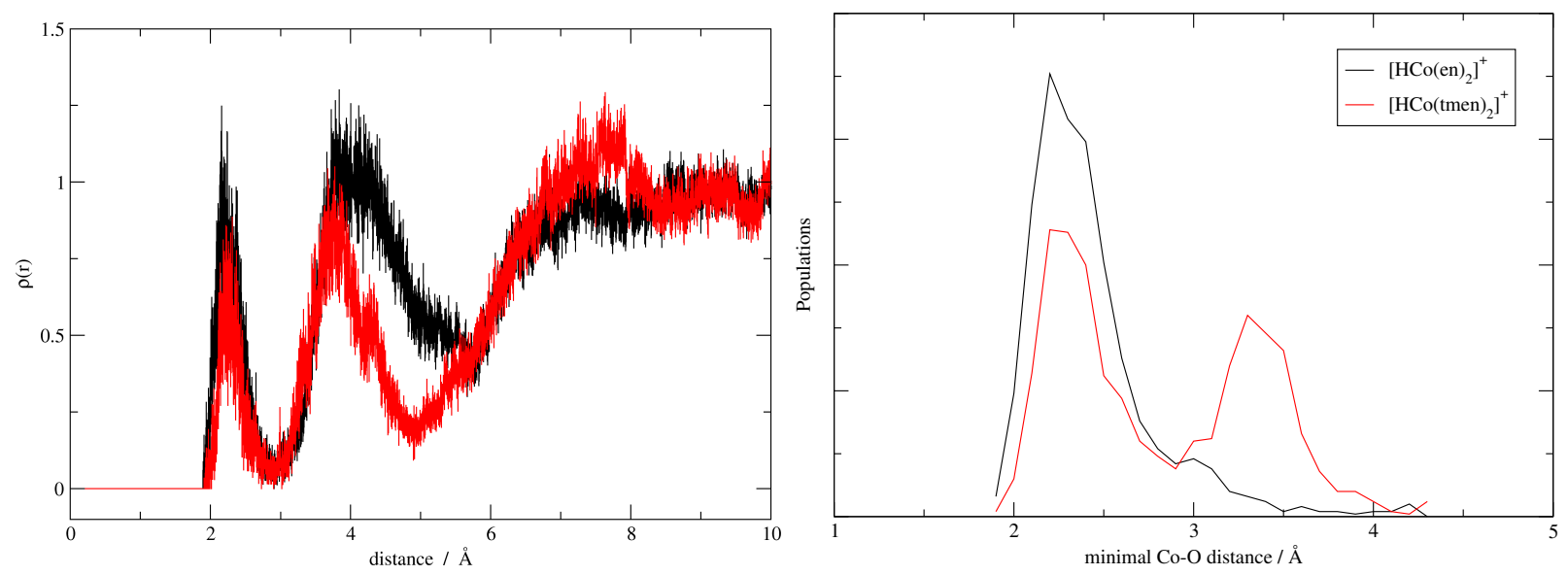

Figure 7: Left: Co-O (water) radial distribution functions; right: distributions of the shortest $\mathrm{Co}-\mathrm{O}$ (water) distances. All data calculated from the 20 ps MD trajectories. Black: $\left[\mathrm{HCo}(\mathrm{en})_{2}\right]^{+}$; red: $\left[\mathrm{HCo}(\text { tmen })_{2}\right]^{+}$. 

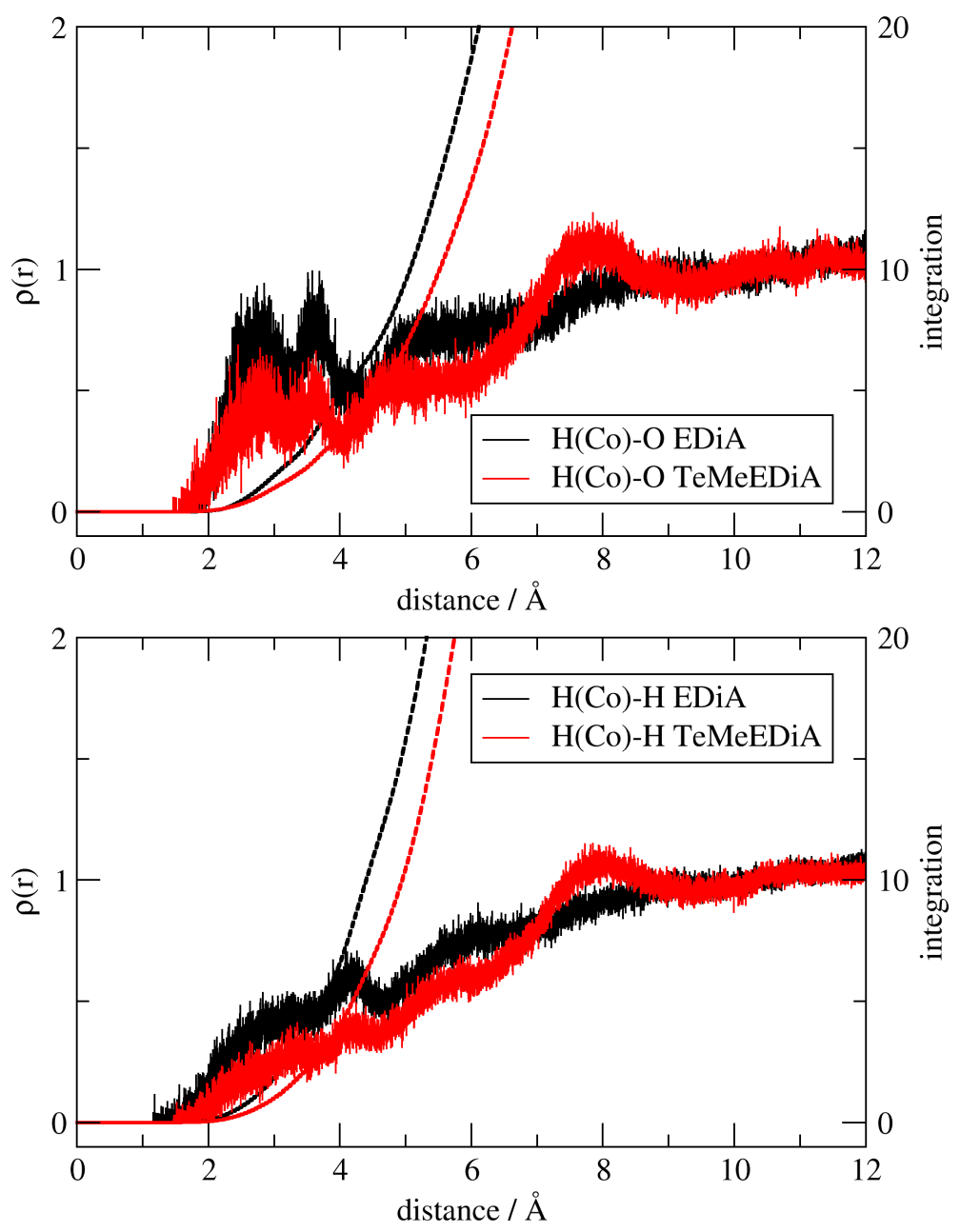

Figure 8: Spherical pairwise radial distribution functions. Top: H (hyd.)-O (water); bottom: H (hyd.) $-\mathrm{H}$ (water). 

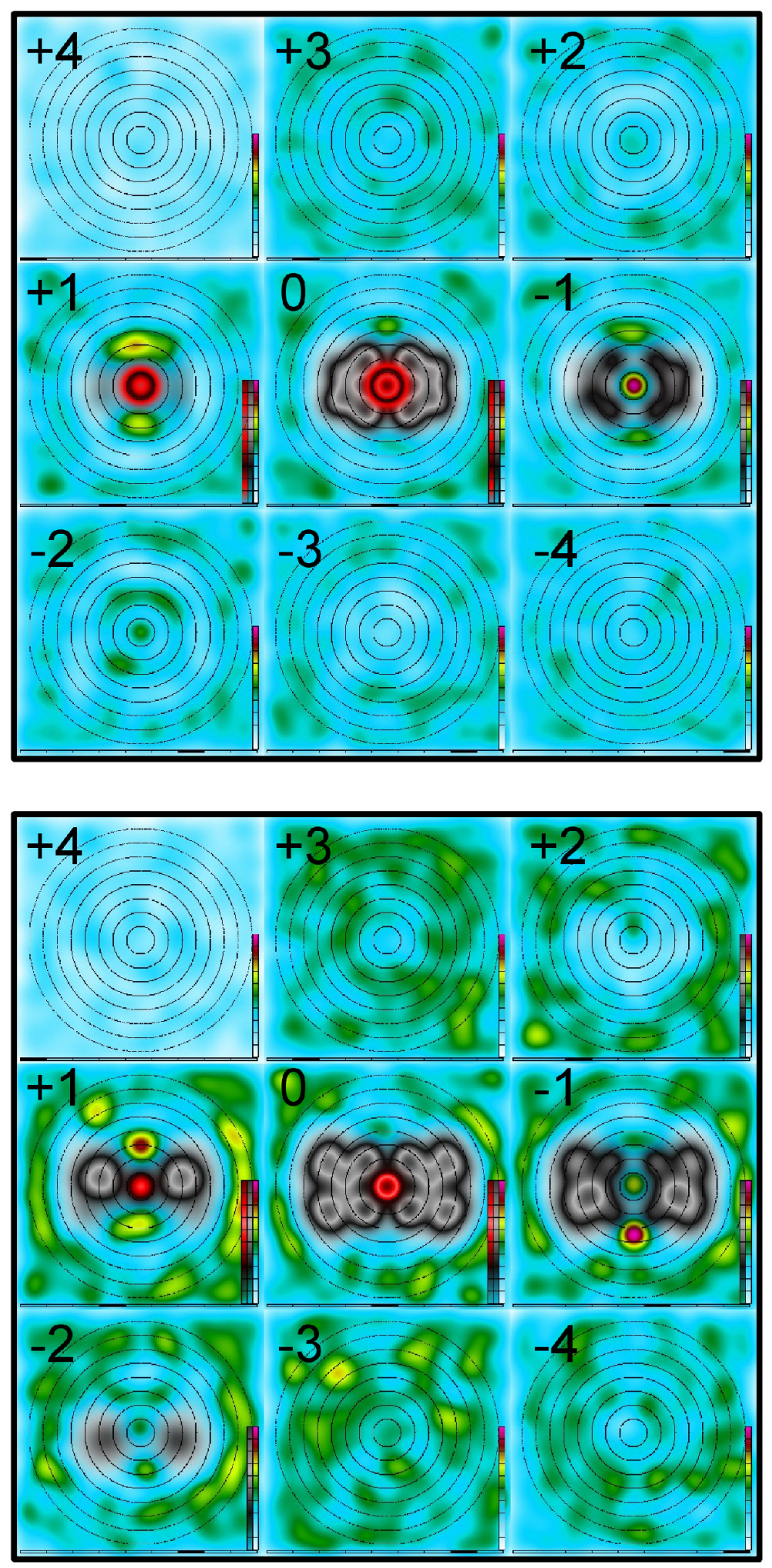

Figure 9: SLICE projection analysis based on superimposed trajectories of the complexes. Top: $\left[\mathrm{HCo}(\mathrm{en})_{2}\right]^{+}$; bottom: $\left[\mathrm{HCo}(\text { tmen })_{2}\right]^{+}$. The three colorbars are as follows: blue/green - water oxygens; gray - complex; red - hydride. 

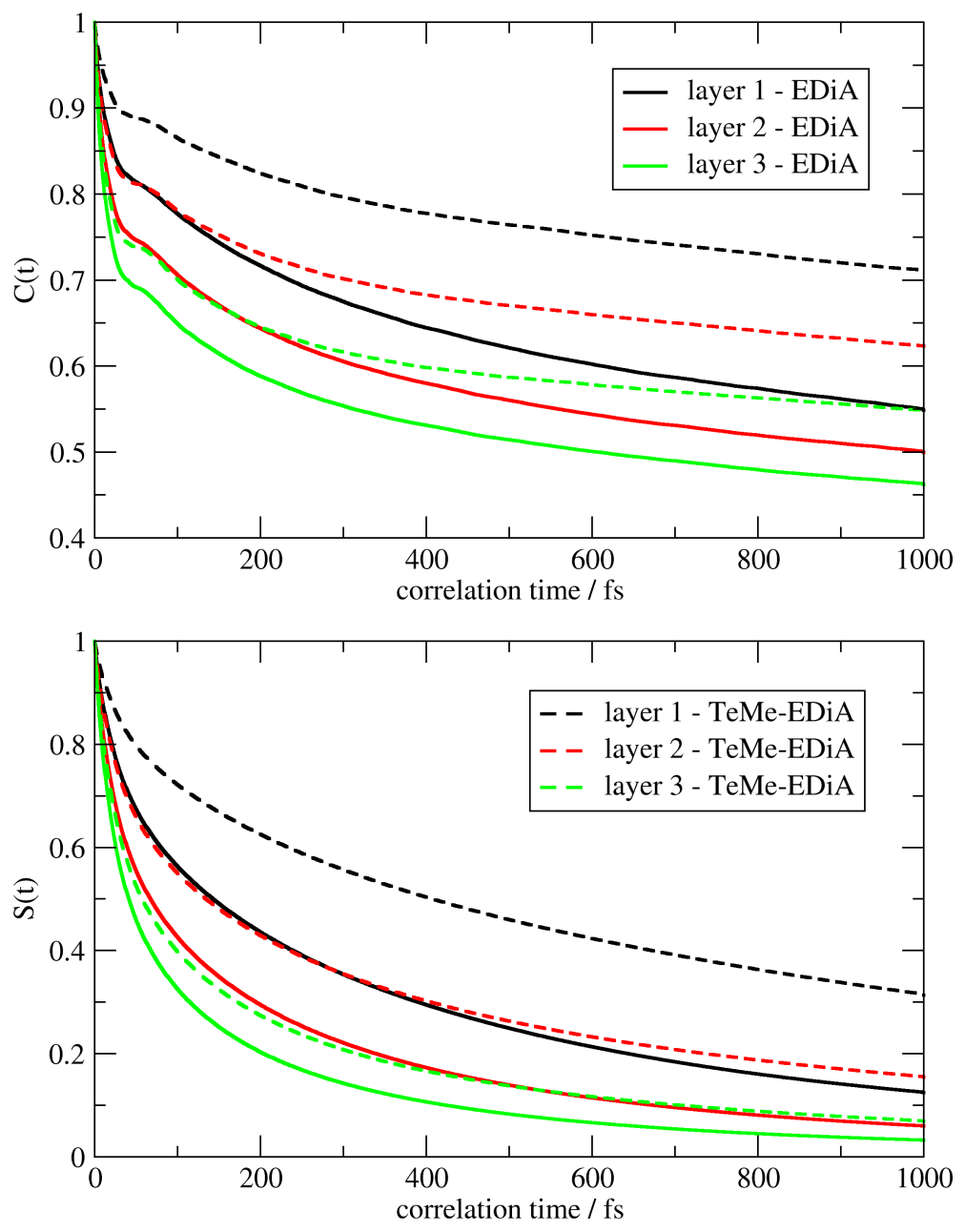

Figure 10: $C(t)$ and $S(t)$ residence time correlation functions of water molecules within individual hydration layers based on a Laguerre tessellation. Full lines: $\left[\mathrm{HCo}(\mathrm{en})_{2}\right]^{+}$; dotted lines: $\left[\mathrm{HCo}(\text { tmen })_{2}\right]^{+}$. Black, red and green refer to hydration layers 1, 2 and 3, respectively. 


\section{Graphical TOC Entry}

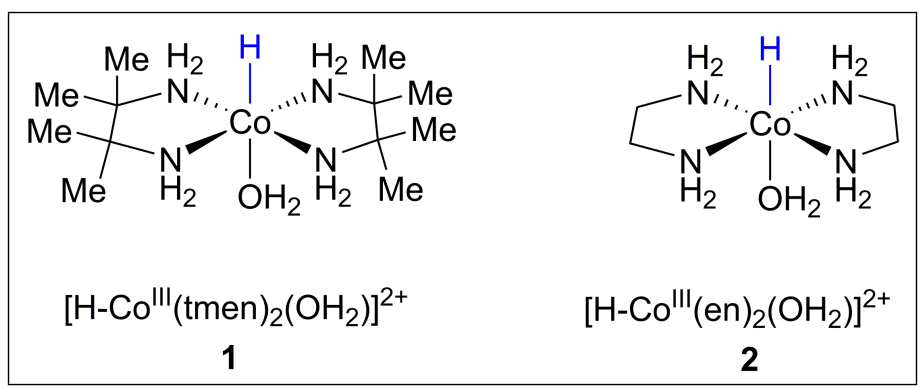

Adaptive QC/MM molecular dynamics simulations have been performed of two models of the cobalt(III)-hydride intermediate that is important in the catalytic cycles of hydrogen evolution mediated by cobaloximes. 